\title{
Micropillar Resonators for Optomechanics in the Extremely High 19-95-GHz Frequency Range
}

\author{
S. Anguiano, ${ }^{1}$ A. E. Bruchhausen, ${ }^{1}$ B. Jusserand, ${ }^{2}$ I. Favero, ${ }^{3}$ F. R. Lamberti, ${ }^{3,4}$ L. Lanco, ${ }^{4}$ I. Sagnes, ${ }^{4}$ A. Lemaître, ${ }^{4}$ \\ N. D. Lanzillotti-Kimura, ${ }^{4}$ P. Senellart, ${ }^{4, *}$ and A. Fainstein ${ }^{1, *}$ \\ ${ }^{1}$ Centro Atómico Bariloche \& Instituto Balseiro, C.N.E.A., CONICET, 8400 San Carlos de Bariloche, Río Negro, Argentina \\ ${ }^{2}$ Institut des NanoSciences de Paris, UMR 7588 C.N.R.S.-Université Pierre et Marie Curie, 75015 Paris, France \\ ${ }^{3}$ Matériaux et Phénomènes Quantiques, Université Paris Diderot, CNRS-UMR 7162, Sorbonne Paris Cité, \\ 10 rue Alice Domon et Léonie Duquet, 75013 Paris, France \\ ${ }^{4}$ Centre de Nanosciences et de Nanotechnologies, C.N.R.S., Université Paris-Sud, \\ Université Paris-Saclay, C2N Marcoussis, 91460 Marcoussis, France
}

(Received 21 December 2016; published 30 June 2017)

\begin{abstract}
Strong confinement, in all dimensions, and high mechanical frequencies are highly desirable for quantum optomechanical applications. We show that GaAs/AlAs micropillar cavities fully confine not only photons but also extremely high frequency (19-95 GHz) acoustic phonons. A strong increase of the optomechanical coupling upon reducing the pillar size is observed, together with record room-temperature $Q$-frequency products of $10^{14}$. These mechanical resonators can integrate quantum emitters or polariton condensates, opening exciting perspectives at the interface with nonlinear and quantum optics.
\end{abstract}

DOI: 10.1103/PhysRevLett.118.263901

Operating mechanical devices at their quantum limit opens interesting perspectives not only in ultrasensitive mass and force sensing but also in the wider field of quantum technologies. Coupling trapped ions to a common vibrational mode has allowed entangling long chains of quantum bits [1]. Ensembles of cold atoms have been used to emulate a macroscopic optomechanical system [2], and hybrid resonators combining quantum electrodynamics and optomechanics have been theoretically predicted to evolve to the single atom limit in the near future [3]. Dynamical backaction of large coherent states of photons has been successfully used to cool mechanical resonators down to their quantum ground state of motion [4-8]. However, the starting bath temperature, which must guarantee a small enough number of excitations in the mechanical mode to start with, scales with the resonator frequency. It is typically of a few microkelvins for a $\mathrm{MHz}$ resonator [6,8], and of $10 \mathrm{~K}$ for the $\mathrm{GHz}$ frequencies attained today by microdisk and optomechanical crystals [9-12]. Reaching higher mechanical frequencies, up to the extremely high frequency range ( $\sim 100 \mathrm{GHz})$, has been hampered so far by top-down nanofabrication techniques such as lithography, as well as the lack of suitable detection methods for the associated fast and minute mechanical motions. Our work shows that this new frequency range is now available in optomechanical resonators with three dimensional optical and mechanical confinement that allow for the integration of single quantum emitters, opening the way to observing novel quantum optomechanical phenomena at high temperatures.

Semiconductor microcavities, based on alternating layers of GaAs and AlAs and etched into micropillar shapes are well known optical resonators [13]. The high refractive index contrast between GaAs and AlAs allows defining optical
Bragg mirrors surrounding a cavity spacer, confining light in one direction. Confinement in the two other directions results from high refractive index contrast between the semiconductor and air. These cavities offer strong flexibility in their design: the optical quality factor can be adjusted through the number of layers in the mirrors and the optical mode volume through the spacer thickness and pillar lateral size. They have been successfully used to obtain state of the art single photon sources [14,15], polariton condensates [16], and to implement quantum simulations [17]. GaAs and AlAs also show a strong acoustic impedance contrast, a property that has been used to fabricate acoustic mirrors. Benefiting from the atomic layer deposition accuracy of epitaxial growth, one-dimensional acoustic cavities have been fabricated in the $\mathrm{GHz}$ to $\mathrm{THz}$ range $[18,19]$. Demonstrating a threedimensional confinement of acoustic phonons in this platform would open exciting perspectives for research at the interface with nonlinear and quantum optics.

In the present work, we demonstrate full three-dimensional confinement for acoustic phonons in GaAs/AlAs micropillar cavities. We implement a pump-probe differential reflectivity measurement that allows measuring the dynamical optomechanical response at high frequency (up to $\mathrm{THz}$ ). The pillars are designed to operate at $880 \mathrm{~nm}$ for the optical mode, close to the GaAs energy gap at room temperature to enhance the optomechanical response [20]. Confined acoustic modes are observed at 19, 58 , and $95 \mathrm{GHz}$ with room temperature mechanical quality factors in the 200 to 1600 range. The three dimensional confinement leads to a strong increase of the optomechanical response when reducing the pillar size.

We study micropillar cavities made from a planar cavity consisting of a $\lambda / 2$ GaAs spacer with $\lambda=880 \mathrm{~nm}$, 
sandwiched between $\mathrm{Ga}_{0.9} \mathrm{Al}_{0.1} \mathrm{As} / \mathrm{Ga}_{0.05} \mathrm{Al}_{0.95} \mathrm{As} \lambda / 4$ distributed Bragg reflectors (DBR). Because of the peculiar material properties of GaAs and AlAs, a planar optical microcavity is also an acoustic cavity confining phonons of the same wavelength [18]. Considering the difference between light and sound speeds, at $\lambda=880 \mathrm{~nm}$ the corresponding fundamental mode for the acoustic cavity is around $19 \mathrm{GHz}$. To obtain the microresonators, the planar cavity is etched into circular or square micropillars with lateral sizes ranging from 50 to $1 \mu \mathrm{m}$, as shown in Fig. 1(a). The results presented in this Letter correspond to square pillars such as the one presented in the figure inset. The three dimensional confinement of the optical field [21] is evidenced by a photoluminescence $k$-space mapping of the modes, as illustrated for a $8 \mu \mathrm{m}$ pillar in Fig. 1(b). Lateral confinement leads to a discretization of the parabolic dispersion of the cavity mode, with mode spacing increasing when reducing the pillar lateral size [13].

In such micropillar structures, a three dimensional mechanical confinement is also expected from the boundary conditions at the pillar edge, where $\overline{\bar{\sigma}} \cdot \vec{n}=\overrightarrow{0}$, with $\overline{\bar{\sigma}}$ the stress tensor and $\vec{n}$ the normal to the surface. Figure 1(c) shows a numerical simulation of a mechanical mode around $19 \mathrm{GHz}$ (corresponding to a breathing of the cavity spacer along $z$ ) calculated for a $3 \mu \mathrm{m}$ circular pillar, where the absolute value of the volumetric strain $|d V / V|$ shows a strong three dimensional confinement.

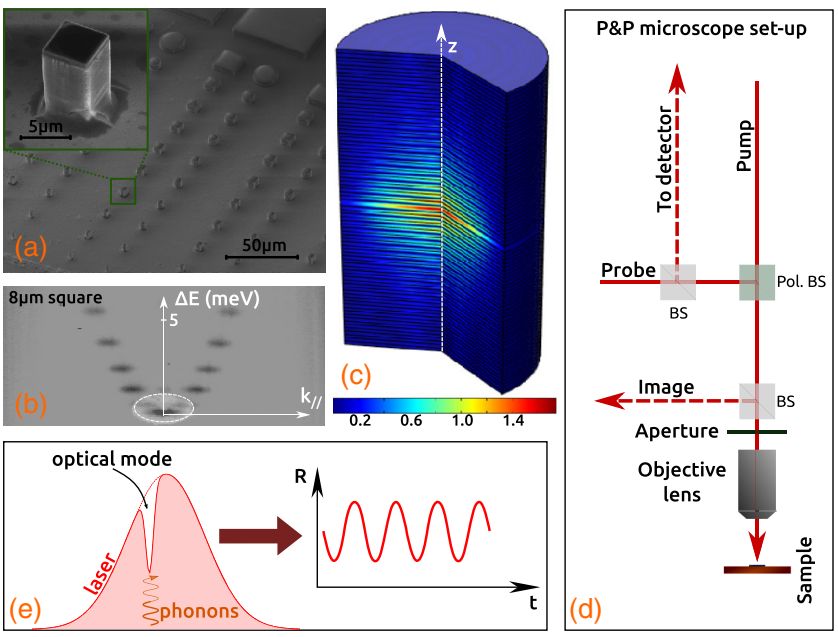

FIG. 1. (a) SEM images of an array of circular and square pillars with lateral sizes ranging from 50 to $1 \mu \mathrm{m}$. The inset presents a zoom on a $5 \mu \mathrm{m}$ square pillar. (b) $k$-space image of the optical cavity modes for a square pillars of $8 \mu \mathrm{m}$ lateral size. The shaded ellipse represents the profile (energy broadening and angular dispersion) of the pump and probe laser pulses. (c) Spatial distribution of $|d V / V|$ associated with a confined mechanical mode around $19 \mathrm{GHz}$, calculated using finite element methods. (d) Scheme of the ultrafast resonant laser microspectroscopy setup. (e) Scheme of the process leading to mechanical signals in reflectance difference time resolved spectroscopy.
To experimentally probe these high frequency confined acoustic modes, standard motion measurement techniques used in cavity optomechanics are not readily available. We implement a time-resolved differential optical reflectivity (pump-probe) measurement [22,23] with micrometer lateral spatial resolution. The resolution is $\sim 4 \mu \mathrm{m}$, implying that single pillars are addressed in the experiments. A scheme of the setup is presented in Fig. 1(d). A pump picosecond optical pulse generates coherent mechanical vibrations. The changes induced on the shape and the indices of refraction of the micropillar are then probed by measuring the reflectivity of a delayed second pulse. Figure 1(e) is a scheme of the spectrum of the incoming probe beam (thin line), with a spectral linewidth of $\sim 1.5 \mathrm{meV}$ exceeding the optical mode linewidth (about $\sim 0.7 \mathrm{meV}$ ). The red shaded curve illustrates the spectrum of the reflected probe beam, where the cavity mode is evidenced as a dip that is modulated at the mechanical frequency. By changing the delay between the pump and probe pulses, we reconstruct the time evolution of the optical reflectivity modulated by the mechanical vibrations.

Two main effects contribute to the optical modulation induced by the pump pulse. First, an electronic contribution arising from two-photon and free carrier absorption leading to a very fast modification of the GaAs index of refraction, blueshifting the optical cavity mode by a few meV. Equilibrium is recovered through carrier recombination typically within 2-5 ns [24]. Second, a mechanical contribution, resulting from coherent mechanical excitations generated by the photoexcited carriers through the deformation potential mechanism [25]. These coherent vibrations lead to a displacement of the interfaces, and modulate the index of refraction through the photoelastic mechanism $[18,26,27]$.

Figure 2 presents a typical signal measured on a $5 \mu \mathrm{m}$ pillar. The top panel displays the as-measured reflectance difference as a function of the delay $t$ between the pump and probe pulses. This signal is dominated by the electronic contribution at short delay and by the mechanical one at long time delays. The middle panel shows the mechanical signal obtained by filtering out the slowly varying spectral components (frequencies smaller than $5 \mathrm{GHz}$ ) and leaving only frequencies up to $100 \mathrm{GHz}$ characteristic of the mechanical contributions. The time dependent spectrum of the mechanical signals, shown in the bottom panel as a color intensity map, is obtained from the windowed Fourier transform calculated using 1000 ps windows with the center spanning from $t=500$ to 4500 ps. Below $2 \mathrm{~ns}$, the electronic excitation leads to a complex intermixed behavior between mechanical vibrations and the optical mode shift. We focus on the delays above $2 \mathrm{~ns}$, where the modulation is dominated by the coherent mechanical vibrations as clearly seen on the bottom panel of Fig. 2 .

The spectrum of a $5 \mu \mathrm{m}$ square pillar obtained from the Fourier transform between 2 and 10 ns is shown in Fig. 3(a). 


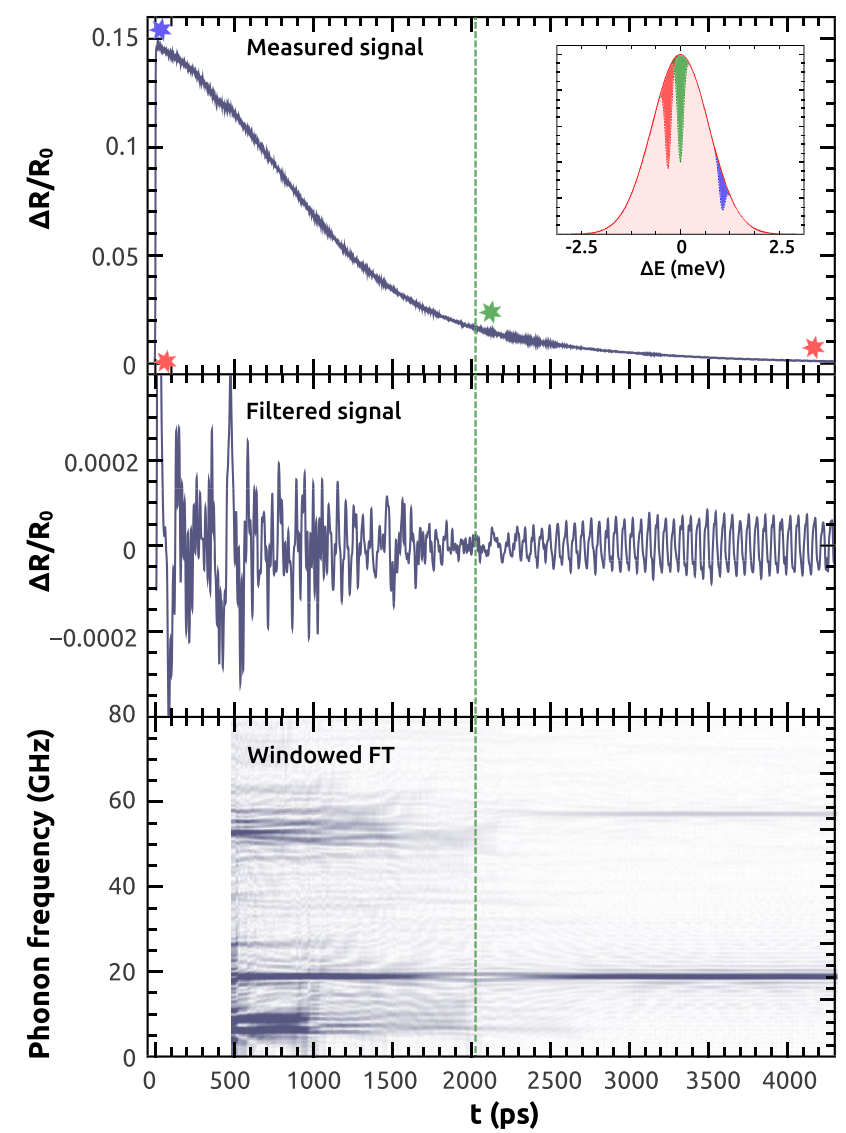

FIG. 2. Top: As-measured differential reflectance time trace for a $5 \mu \mathrm{m}$ pillar, varying the delay $t$ between the pump and probe pulses. The inset is a schematic representation of the laser pulse energy distribution, and the cavity mode (dip in the reflected probe) at different times after pump excitation. The time before (after) the arrival of the pump is indicated with the initial red(blue) star and dip. Excited carrier relaxation leads to the recovery of the cavity mode, which around 2000 ps passes through the central energy of the laser (vertical dashed line, green star, and dip). At longer times the equilibrium situation is recovered (final red star and dip). Middle: filtered time trace corresponding to frequencies between 5 and $100 \mathrm{GHz}$. Bottom: windowed Fourier transform (WFT) of the filtered trace, obtained with 1000 ps windows.

Three mechanical resonances are observed at $\sim 19, \sim 58$, and $\sim 95 \mathrm{GHz}$. Because the pump induced perturbation is concentrated in the GaAs-spacer layer, the generated localized strain mainly corresponds to vibrations vertically confined in the cavity. The observed resonances correspond to the fundamental mode, and the third and fifth overtones. The absence of even order gaps for $\lambda / 4, \lambda / 4$ mirrors prevents the existence of even order acoustic modes confined in the vertical direction.

The mechanical signal observed at each frequency $\sim 19, \sim 58$, and $\sim 95 \mathrm{GHz}$ actually corresponds to a group of fully confined mechanical resonances. Indeed, like for the optical field, several mechanical modes with different lateral profiles arise from the three-dimensional confinement. Moreover, a blueshift of each of these mechanical
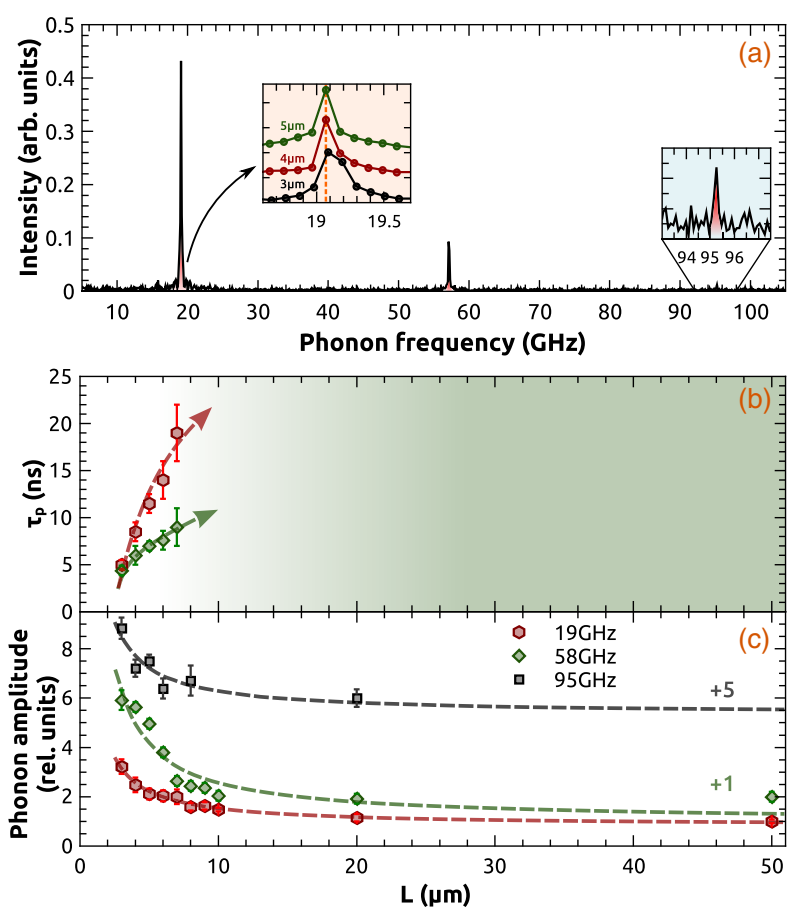

FIG. 3. (a) Vibrational spectra of a $5 \mu \mathrm{m}$ pillar obtained through the Fourier transform of the oscillating part extracted from the reflectance difference signal. The fundamental cavity mode at approximately $19 \mathrm{GHz}$ and two overtones at 58 and $95 \mathrm{GHz}$ are highlighted. The inset compares the $19 \mathrm{GHz}$ mode of pillars of 3, 4, and $5 \mu \mathrm{m}$ size. (b) Lifetime of the 19 and $58 \mathrm{GHz}$ cavity confined mechanical vibration as a function of pillar size $L$. Error bars reflect the measured amplitude uncertainty. The colored region marks the resolution limit of our pump and probe technique. (c) Mechanical signal amplitude $\left(\Delta R / R_{0}\right)$ corresponding to the three confined acoustic modes. Values are normalized to the amplitude observed for the $50 \mu \mathrm{m}$ pillar, and have been vertically shifted for clarity. Dashed curves are fits with a $1 / L$ dependence (see text for details).

resonances is expected when reducing the pillar size. Both mode frequency spacing and blueshift are expected to be around $40-60 \mathrm{MHz}$ for a 3 micron-size pillar, which is the smallest size we can study with our present spatial resolution. This is slightly below our spectral resolution of $100 \mathrm{MHz}$, which is Fourier-transform limited by the length of the multiple-pass delay line used. Note that cross talk in degenerate pump-probe experiments makes measurement of vibrational signals in pillars smaller than 2 microns very challenging. Notwithstanding these limitations, clear signatures of mode shift and transverse level splitting (appearing as a high-energy asymmetry) can be consistently and reproducibly identified in the vibrational spectra [see the inset for the $20 \mathrm{GHz}$ mode in Fig. 3(a)]. To provide further evidence of the lateral confinement of the mechanical modes, we study next both their lifetime and optomechanical signal intensity.

Figure 3(b) shows the lifetime of the 19 and $58 \mathrm{GHz}$ cavity confined acoustic vibration as a function of pillar 
size $L$. The lifetime dependence cannot be accessed for the modes around $95 \mathrm{GHz}$ due to limited signal intensity. The colored region marks the present resolution limit of our pump-probe technique. The results indicate that the lifetimes for the $19 \mathrm{GHz}$ mode are at least of $20 \mathrm{~ns}$ for pillar lateral sizes larger than $6 \mu \mathrm{m}$. Such observation shows the high quality of the fabricated resonators since this value is close to the phonon lifetime in the GHz range studied in bulk GaAs/AlAs structures at room temperature. For pillar sizes below $7 \mu \mathrm{m}$ a decrease of the mechanical mode lifetime is observed down to $5 \mathrm{~ns}$ for a pillar of $3 \mu \mathrm{m}$. As also observed for confined photons [28], phonon losses induced by lateral surfaces are expected to become more relevant for smaller devices because of an increased surface to volume ratio. The phonon lifetime is lower at $58 \mathrm{GHz}$ than at $19 \mathrm{GHz}$ for all pillar sizes, presumably due to phonon intrinsic anharmonicity. Indeed, phonons can decay into two or more phonons, or can scatter with background phonons. These processes are not significant at $\mathrm{MHz}$ frequencies, but become relevant in the $\mathrm{GHz}-\mathrm{THz}$ range at room temperature [19]. Since in principle we do not couple to a single mechanical mode but rather to a group of unresolved transverse modes, mode dephasing could also be contributing to the observed signal decay. Thus, the measured lifetimes can be considered a lower bound to the mechanical $Q$ factor; see Supplemental Material [29].

We now study the dependence of the mechanical response intensity with pillar size. The intensity of the signal scales as the optomechanical coupling factor $g_{0}$ which determines the net effect a single phonon produces on the optical cavity resonance. The phonon generation process saturates with the incident power: the pump laser intensity is set beyond this saturation so that the optical force does not depend on the pump power and can be assumed as the same in all cases. The intrinsic optomechanical response then scales as the reflected probe intensity $\Delta R$ normalized to the reflected probe power, $R_{0}$ [29]. The absolute mechanical response for a given pillar size decreases when going from 19 to $95 \mathrm{GHz}$, similar to what is observed for a planar cavity [see Fig. 3(a)]. Indeed, this behavior is mostly dominated by the $z$ dependence of the optomechanical coupling factor [18]. What is more relevant here is the optomechanical response for the three confined modes as a function of the pillar size, as shown in Fig. 3(c). For all frequencies, a strong increase of the mechanical signal with decreasing pillar size is observed. The optomechanical coupling is related to the overlap between the optical and mechanical modes. Because the amplitude of confined modes scale as $1 / \sqrt{V}$ ), with $V$ the mode volume, it can be shown that the optomechanical coupling should scale as $1 / L$ when the pillar lateral area shrinks if both optical and mechanical modes are confined [29]. This behavior well accounts for the observed dependence as illustrated with the dashed curves in Fig. 3(c). We note that a rough theoretical estimation of the optomechanical coupling factor $g_{0} / 2 \pi$, assuming a nonresonant photoelastic mechanism, leads to values close to the $\mathrm{MHz}$ range. This implies that far from the gap, where, for example, quantum dots could be excited, the system remains in a weak coupling regime.

The strong dependence of the mechanical signal as well as of the mechanical $Q$ factor with the pillar diameter provide strong evidence for the three dimensional confinement of the mechanical modes in the micropillars. The measured lifetimes correspond to room temperature mechanical $Q$ factors between 200 and 1000 for the $19 \mathrm{GHz}$ mode, and between 750 and 1600 for the $58 \mathrm{GHz}$ mode. These numbers result in $Q$-frequency products between $10^{12}$ and $10^{14}$ that approach state of the art values and, to the best of our knowledge, are record at room temperature, showing the strong potential of this new optomechanical platform [4]. In practice, this implies that about 100 coherent quantum control operations could be performed on this mechanical mode at room temperature.

We finally briefly discuss the complex dynamics observed at shorter times in Fig. 2. Pulsed studies in cavity optomechanics have only been recently reported to access the transient backaction dynamics [32]. Insight on such dynamics with picosecond time resolution can be grasped from the traces displayed in the middle and bottom panels of Fig. 2 . Complex multiple frequency spectra are observed, with additional frequencies observed around 19 and $58 \mathrm{GHz}$. The same systematics is observed in all the studied pillars immediately after pump excitation. These observations hint towards a form of nonlinear optomechanical backaction. Indeed, the standard radiation pressure optomechanical coupling itself is nonlinear, and has been shown to lead to frequency doubling, multistabilities [33,34], and chaotic behavior $[35,36]$. At the shorter times where the complex dynamics is observed, the laser detuning varies on a time scale itself commensurable with mechanical periods [37]. Advanced modeling, beyond existing models of optomechanics, is required to account for these observations.

Our work shows that a new frequency range is now available in three dimensionally confined optomechanical resonators, opening the way to observing quantum optomechanics phenomena at high temperature. The studied micropillar structures can naturally integrate semiconductor quantum wells [38] or be optimally coupled to single quantum dots [39]. The acoustic manipulation and control of interactions involving polaritons [38] and quantum emitters, and the possibility of cooling and control of quantum mechanical states at the single photon level [3], are only examples of the exciting prospects offered by this new optomechanical platform.

This work was partially supported by the ANPCyT Grants No. 2012-1661 and No. 2013-2047, the ERC Starting Grant No. 277885 QD-CQED, the French Agence Nationale pour la Recherche (grant ANR 
QDOM) the French RENATECH network, a public grant overseen by the French National Research Agency (ANR) as part of the "Investissements d'Avenir" program (Labex NanoSaclay, reference: ANR-10-LABX-0035), and the international franco-argentinean laboratory LIFAN (CNRS-CONICET). N. D. L. K. was supported by the FP7 Marie Curie Fellowship OMSiQuD.

*Corresponding author.

pascale.senellart-mardon@c2n.upsaclay.fr

${ }^{\dagger}$ Corresponding author.

afains@cab.cnea.gov.ar

[1] R. Blatt and C. F. Roos, Quantum simulations with trapped ions, Nat. Phys. 8, 277 (2012).

[2] T. P. Purdy, D. W. C. Brooks, T. Botter, N. Brahms, Z.-Y. Ma, and D. M. Stamper-Kurn, Tunable Cavity Optomechanics with Ultracold Atoms, Phys. Rev. Lett. 105, 133602 (2010).

[3] J. Restrepo, C. Ciuti, and I. Favero, Single-Polariton Optomechanics, Phys. Rev. Lett. 112, 013601 (2014).

[4] M. Aspelmeyer, T. J. Kippenberg, and F. Marquardt, Cavity optomechanics, Rev. Mod. Phys. 86, 1391 (2014).

[5] A. D. O'Connell, M. Hofheinz, M. Ansmann, R. C. Bialczak, M. Lenander, E. Lucero, M. Neeley, D. Sank, H. Wang, M. Weides, J. Wenner, J. M. Martinis, and A. N. Cleland, Quantum ground state and single-phonon control of a mechanical resonator, Nature (London) 464, 697 (2010).

[6] J. D. Teufel, T. Donner, D. Li, J. H. Harlow, M. S. Allman, K. Cicak, A. J. Sirois, J. D. Whittaker, K. W. Lehnert, and R. W. Simmonds, Sideband cooling of micromechanical motion to the quantum ground state, Nature (London) $\mathbf{4 7 5}$, 359 (2011).

[7] J. Chan, T. P. M, Alegre, A. H. Safavi-Naeini, J. T. Hill, A. Krause, S. Groeblacher, M. Aspelmeyer, and O. Painter, Laser cooling of a nanomechanical oscillator into its quantum ground state, Nature (London) 478, 89 (2011).

[8] R. W. Peterson, T. P. Purdy, N. S. Kampel, R. W. Andrews, P. L. Yu, K. W. Lehnert, and C. A. Regal, Laser Cooling of a Micromechanical Membrane to the Quantum Backaction Limit, Phys. Rev. Lett. 116, 063601 (2016).

[9] L. Ding, C. Baker, P. Senellart, A. Lemaitre, S. Ducci, G. Leo, and I Favero, High Frequency GaAs Nano-Optomechanical Disk Resonator, Phys. Rev. Lett. 105, 263903 (2010).

[10] M. Eichenfield, J. Chan, R. M. Camacho, K. J. Vahala, and O. Painter, Optomechanical crystals, Nature (London) 462 , 78 (2009).

[11] X. Sun, X. Zhang, and H. X. Tang, High-Q silicon optomechanical microdisk resonators at gigahertz frequencies, Appl. Phys. Lett. 100, 173116 (2012).

[12] R. Van Laer, B. Kuyken, D. Van Thourhout, and R. Baets, Interaction between light and highly confined hypersound in a silicon photonic nanowire, Nat. Photonics 9, 199 (2015).

[13] J. M. Gérard, D. Barrier, J. Y. Marzin, R. Kuszelewicz, L. Manin, E. Costard, V. Thierry-Mieg, and T. Rivera, Quantum boxes as active probes for photonic microstructures: The pillar microcavity case, Appl. Phys. Lett. 69, 449 (1996).

[14] N. Somaschi, V. Giesz, L. De Santis, J. C. Loredo, M. P. Almeida, G. Hornecker, S. L. Portalupi, T. Grange, C. Antón,
J. Demory, C. Gómez, I. Sagnes, N. D. Lanzillotti-Kimura, A. Lemaître, A. Auffeves, A. G. White, L. Lanco, and P. Senellart, Near-optimal single-photon sources in the solid state, Nat. Photonics 10, 340 (2016).

[15] X. Ding, Y. He, Z.-C. Duan, N. Gregersen, M.-C. Chen, S. Unsleber, S. Maier, C. Schneider, M. Kamp, S. Höfling, C.-Y. Lu, and J.-W. Pan, On-Demand Single Photons with High Extraction Efficiency and Near-Unity Indistinguishability from a Resonantly Driven Quantum Dot in a Micropillar, Phys. Rev. Lett. 116, 020401 (2016).

[16] D. Bajoni, P. Senellart, E. Wertz, I. Sagnes, A. Miard, A. Lemaître, and J. Bloch, Polariton Laser Using Single Micropillar GaAs-GaAlAs Semiconductor Cavities, Phys. Rev. Lett. 100, 047401 (2008).

[17] T. Jacqmin, I. Carusotto, I. Sagnes, M. Abbarchi, D. D. Solnyshkov, G. Malpuech, G. Galopin, A. Lemaître, J. Bloch, and A. Amo, Direct Observation of Dirac Cones and a Flatband in a Honeycomb Lattice for Polaritons, Phys. Rev. Lett. 112, 116402 (2014).

[18] A. Fainstein, N. D. Lanzillotti-Kimura, B. Jusserand, and B. Perrin, Strong Optical-Mechanical Coupling in a Vertical GaAs/AlAs Microcavity for Subterahertz Phonons and Near-Infrared Light, Phys. Rev. Lett. 110, 037403 (2013).

[19] G. Rozas, M. F. P. Winter, B. Jusserand, A. Fainstein, B. Perrin, E. Semenova, and A. Lemaitre, Lifetime of $\mathrm{THz}$ Acoustic Nanocavity Modes, Phys. Rev. Lett. 102, 015502 (2009).

[20] B. Jusserand, A. N. Poddubny, A. V. Poshakinskiy, A. Fainstein, and A. Lemaitre, Polariton Resonances for Ultrastrong Coupling Cavity Optomechanics in GaAs/AlAs Multiple Quantum Wells, Phys. Rev. Lett. 115, 267402 (2015).

[21] J. M. Gérard, B. Sermage, B. Gayral, B. Legrand, E. Costard, and V. Thierry-Mieg, Enhanced Spontaneous Emission by Quantum Boxes in a Monolithic Optical Microcavity, Phys. Rev. Lett. 81, 1110 (1998).

[22] C. Thomsen, H. T. Grahn, H. J. Maris, and J. Tauc, Surface generation and detection of phonons by picosecond light pulses, Phys. Rev. B 34, 4129 (1986).

[23] A. Bartels, T. Dekorsy, H. Kurz, and K. Koehler, Coherent Zone-Folded Longitudinal Acoustic Phonons in Semiconductor Superlattices: Excitation and Detection, Phys. Rev. Lett. 82, 1044 (1999).

[24] H. Thyrrestrup, E. Yüce, G. Ctistis, J. Claudon, W. L. Vos, and J. M. Gérard, Differential ultrafast all-optical switching of the resonances of a micropillar cavity, Appl. Phys. Lett. 105, 111115 (2014).

[25] P. Ruello and V. E. Gusev, Physical mechanisms of coherent acoustic phonons generation by ultrafast laser action, Ultrasonics 56, 21 (2015).

[26] C. Baker, W. Hease, D.-T. Nguyen, A. Andronico, S. Ducci, G. Leo, and I. Favero, Photoelastic coupling in gallium arsenide optomechanical disk resonators, Opt. Express 22, 14072 (2014).

[27] P. T. Rakich, C. Reinke, R. Camacho, P. Davids, and Z. Wang, Giant Enhancement of Stimulated Brillouin Scattering in the Subwavelength Limit, Phys. Rev. X 2, 011008 (2012).

[28] T. Rivera, J.-P. Debray, J. M. Gérard, B. Legrand, L. ManinFerlazzo, and J. L. Oudar, Optical losses in plasma-etched 
AlGaAs microresonators using reflection spectroscopy, Appl. Phys. Lett. 74, 911 (1999).

[29] See Supplemental Material at http://link.aps.org/ supplemental/10.1103/PhysRevLett.118.263901 for details of the samples, the experimental setup, and the experimental procedures used to determine mechanical mode lifetimes and amplitudes, which includes Ref. [30,31].

[30] P. Sesin, P. Soubelet, V. Villafañe, A. E. Bruchhausen, B. Jusserand, A. Lemaitre, N. D. Lanzillotti-Kimura, and A. Fainstein, Dynamical optical tuning of the coherent phonon detection sensitivity in DBR-based GaAs optomechanical resonators, Phys. Rev. B 92, 075307 (2015).

[31] A. Bruchhausen, R. Gebs, F. Hudert, D. Issenmann, G. Klatt, A. Bartels, O. Schecker, R. Waitz, A. Erbe, E. Scheer, J.-R. Huntzinger, A. Mlayah, and T. Dekorsy, Subharmonic Resonant Optical Excitation of Confined Acoustic Modes in a Free-Standing Semiconductor Membrane at GHz Frequencies with a High-Repetition-Rate Femtosecond Laser, Phys. Rev. Lett. 106, 077401 (2011).

[32] S. M. Meenehan, J. D. Cohen, G. S. MacCabe, F. Marsili, M. D. Shaw, and O. Painter, Pulsed Excitation Dynamics of an Optomechanical Crystal Resonator near Its Quantum Ground State of Motion, Phys. Rev. X 5, 041002 (2015).

[33] F. Marquardt, J. G. E. Harris, and S. M. Girvin, Dynamical Multistability Induced by Radiation Pressure in HighFinesse Micromechanical Optical Cavities, Phys. Rev. Lett. 96, 103901 (2006).

[34] C. Metzger, M. Ludwig, C. Neuenhahn, A. Ortlieb, I. Favero, K. Karrai, and F. Marquardt, Self-Induced Oscillations in an
Optomechanical System Driven by Bolometric Backaction, Phys. Rev. Lett. 101, 133903 (2008).

[35] T. Carmon, H. Rokhsari, L. Yang, T. J. Kippenberg, and K. J. Vahala, Temporal Behavior of Radiation-PressureInduced Vibrations of an Optical Microcavity Phonon Mode, Phys. Rev. Lett. 94, 223902 (2005).

[36] T. Carmon, M. C. Cross, and K. J. Vahala, Chaotic Quivering of Micron-Scaled On-Chip Resonators Excited by Centrifugal Optical Pressure, Phys. Rev. Lett. 98, 167203 (2007).

[37] Note that the apparent splitting of the $19 \mathrm{GHz}$ mode at $\sim 2000$ ps (at the position of the vertical dashed line in the bottom panel of Fig. 2) is a mathematical effect of the windowed Fourier transform, arising due to the $\pi$ change of phase of the mechanically induced reflectivity oscillations observed upon crossing of the cavity mode with the maximum of the laser energy. This feature can, in fact, be used as a signature for the dynamical transition of the cavity mode from blueshift to redshift with respect to the laser maximum.

[38] G. Rozas, A. E. Bruchhausen, A. Fainstein, B. Jusserand, and A. Lemaître, Polariton path to fully resonant dispersive coupling in optomechanical resonators, Phys. Rev. B 90, 201302(R) (2014).

[39] A. Dousse, L. Lanco, J. Suffczyński, E. Semenova, A. Miard, A. Lemaître, I. Sagnes, C. Roblin, J. Bloch, and P. Senellart, Controlled Light-Matter Coupling for a Single Quantum Dot Embedded in a Pillar Microcavity Using Far-Field Optical Lithography, Phys. Rev. Lett. 101, 267404 (2008). 